\title{
Model of the Gravimeter Capacitor with Two Dielectrics
}

\author{
Prapat Mahapattanathai, Wipada Ngansom, Kanphot Thongcham and Chamnan Temmuangpuk \\ Faculty of Science, Ramkhamhaeng University, Bangkok 10240, Thailand
}

Received: October 21, 2012 / Accepted: November 26, 2012 / Published: March 25, 2013.

\begin{abstract}
Design for gravimeter capacitor, the first model contains dielectric constant $\kappa$ in partially filled with material and the second contain dielectric constant $\kappa_{1}$ in space between the cylinder is half-filled by a semi-cylindrical. Thus, capacitance varies in accordance with the length of the device in the cylindrical conductor or when the length of the device in the cylindrical capacitor varies in accordance with at various test locations. It can easily be shown that a change in height of approximately $1 \mathrm{~m}$ results in a change in the gravitational acceleration of $\Delta g=-0.0031 \mathrm{~cm} / \mathrm{s}^{2}$ whereas the value of the gravitational acceleration at Ramkhamhaeng university Bangkok, Thailand used as the reference point was equal to $978.310 \mathrm{~cm} / \mathrm{s}^{2}$.
\end{abstract}

Key words: Gravimeter capacitor, electrostatic gravimeter, cylindrical capacitor.

\section{Introduction}

The gravitational attraction of a sphere with central symmetry [1] at a point external to the sphere, is mathematically the same as though the entire mass of the sphere which were concentrated at its centre. In reality, the value of gravitational acceleration $(g)$ is not constant, but rather, changes over surface of the earth. In the field of geology, the value of gravitational acceleration can be used to determine many characteristics of the Earth such as rock density, porosity, layer composition, locations of underground aquifers, caverns and air pockets. By monitoring the slight changes of the value of gravitational acceleration, geologists can extrapolate information about the structure of the Earth's crust below the surface [2]. All methods of extrapolating data from the measurements of gravitational acceleration can be derived from forces of nature which is the attraction between all masses. According to the Newtonian law of gravity [3], the gravitational force between any two point masses is given by:

$$
F=\frac{G M_{E} m}{r^{2}}
$$

Corresponding author: Prapat Mahapattanathai, assistant professor, research field: applied physics. E-mail: P_wipada@hotmail.com.
This force acts in the direction joining the two masses.

The resulting acceleration $(\mathrm{g})$ of mass $m$ is given by:

$$
g=\frac{G M_{E}}{r^{2}}
$$

Where, $M_{E}$ is the mass of the Earth, $G$ is the gravitational constant, and $r$ is the distance from the center of the earth.

This model applies reasonably well to the Earth is essentially a spheroid [3], with a slight flattening at the poles, a mean radius $R_{E}$ of $6368 \mathrm{~km}$ and mass $M_{E}$, of $5.98 \times 10^{24} \mathrm{~kg}$. We find that at surface of the Earth, its mean of gravitation acceleration $(g)$ is given by 9.80 $\mathrm{m} / \mathrm{s}^{2}$. At the equator it reduces to about $9.78 \mathrm{~m} / \mathrm{s}^{2}$, and at the poles it increases to about $9.83 \mathrm{~m} / \mathrm{s}^{2}$, reflecting the fact that one is farther from center of the Earth at equator than at poles. In lieu of using meters per second squared $\left(\mathrm{m} / \mathrm{s}^{2}\right)$ for gravitation acceleration, one often employs unit of Gal, where $1 \mathrm{Gal}=10^{-2} \mathrm{~m} / \mathrm{s}^{2}$.

Current techniques of measuring gravitational acceleration, one method used the free fall method which involves dropping an object of mass, can be measure the time in which it takes the mass to traverse the distance. A second more accurate method of a simple pendulum can be used to determine a much more precise for gravitational acceleration [4]. There 
are some geophysicists that may use a double pendulum gravimeter to conduct preliminary measurements of gravitational acceleration. However, the changes that occur as you move across the surface of the Earth are still not accurate enough to obtain some of the more detailed information about the Earth's crust [5].

The method used most commonly by geophysicists interested in obtaining an accurate measurement of the gravitational acceleration field, requires the analysis of a mass spring system. A mass hanging from a stationary spring is under the influence of the following two forces, the force of gravity pulling it down and the force of the spring pulling it up. These two forces are equal and opposite, causing the mass to be motionless while in a state of static equilibrium. One can obtain by setting the two forces equal to one another and solving for $\mathrm{g}$ as shown below:

$$
\begin{aligned}
& \mathrm{mg}=\mathrm{kx} \\
& \mathrm{g}=\frac{\mathrm{k}}{\mathrm{m}} \mathrm{x}
\end{aligned}
$$

where $\mathrm{k}$ is the spring constant and $x$ is the distance of the spring stretched from its equilibrium position without the mass.

The most difficult aspect in the design of the gravimeter is to determine the changes in the stretch of the spring with slight changes in gravitational acceleration [6]. The purpose of this work is to propose an easier method of measuring the slight changes in the distance $(x)$ used in the mass-spring gravimeter. Our resultants show that with a simple application of elementary electrostatics, the same measurements are possible with a lower cost and a less complicated apparatus. For geologists, this may provide an easy-to-build, inexpensive gravimeter yielding fairly accurate measurements of gravitational acceleration. For college faculty, it will provide an excellent way to demonstrate changes in gravitational acceleration using simple concepts of physics. Our design is called the electrostatic gravimeter, and it uses a capacitor as the primary component in the device for measuring changes in distance $(x)$.
Consideration as far as the cylindrical capacitor two dielectrics for detailed design of the electrostatic gravimeter, we will first review some of the electrostatic theory used to describe capacitors [7]. The first models of our electrostatic gravimeter is a conducting cylinder of a metal pipes radius $a$. It is surrounded by a concentric tube of inner radius $c$. The space between is partially filled (from $b$ out to $c$ ) with material of dielectric constant $\kappa$, given by:

$$
\vec{E}=\frac{\lambda}{2 \pi \varepsilon_{\mathrm{o}} \kappa r} \hat{r}
$$

From

$$
\begin{gathered}
\Delta V=V_{b}-V_{a}=-\int_{a}^{b} \vec{E} \times d \vec{r} \\
\Delta V=\frac{\lambda}{2 \pi \varepsilon_{0}}\left[\ln \left(\frac{b}{a}\right)+\frac{1}{\kappa} \ln \left(\frac{c}{b}\right)\right]
\end{gathered}
$$

From Eq. (6) capacitance is given by:

$$
C=\frac{2 \pi_{o} L}{\ln \left(\frac{b}{a}\right)+\frac{1}{k} \ln \left(\frac{c}{b}\right)}
$$

where $\varepsilon_{0}$ is the electric permittivity of free space, $\kappa$ is the dielectric constant and $L$ is the length of both cylinders.

The second one, a cylindrical capacitor consists of a metal pipes two concentric conducting cylinders of inner and outer radii $a$ and $b$ respectively. The empty space between the cylinders is half-filled by a semi-cylindrical shell of dielectric. The capacitance is given by:

$$
\begin{gathered}
\vec{E}=\frac{\rho_{s} a}{\varepsilon_{o} \kappa r} \hat{r} \\
\Delta V=\frac{\rho_{s} a}{\varepsilon_{o} \kappa} \ln \frac{b}{a}
\end{gathered}
$$

From Eq. (9) capacitance is given by:

$$
\begin{gathered}
C=C_{1}+C_{2}=\frac{\pi \varepsilon_{\mathrm{o}} \kappa_{1} L}{\ln \left(\frac{b}{a}\right)}+\frac{\pi \varepsilon_{\mathrm{o}} \kappa_{2} L}{\ln \left(\frac{b}{a}\right)} \\
C=\frac{2 \pi \varepsilon_{\mathrm{o}} \kappa_{\text {ravg }} L}{\ln \left(\frac{b}{a}\right)}
\end{gathered}
$$


where $\kappa_{2}$ is dielectric constant of free space, $\kappa_{\text {ravg }}=$ $1 / 2\left(\kappa_{1}+\kappa_{2}\right)$, and $L$ is the length of both cylinders.

\section{Experiments}

Our electrostatic gravimeter of both models in this work are shown in Fig. 1a-1b. For the first model (Fig. 1a) contains dielectric constant $\kappa$ in partially filled with material. The second model (Fig. 1b) constant $\kappa_{1}$ in space between the cylinders is half-filled by a semi-cylindrical. The dielectric constant $\kappa$ and $\kappa_{1}$ in audition process are the same type. This instrument allows us to examine the effects of changes in gravitational acceleration on the basis of the relationship between a system of mass springs and capacitance values for the cylindrical capacitor.

In rigorously investigated for gravitational acceleration, we start from obtaining an expression for $L$, thus:

$$
L=h-y_{\mathrm{o}}+x
$$

Representation $x$ equals $\mathrm{mg} / \mathrm{k}$ by using this result substitution into Eq. (11), thus:

$$
L=h-y_{\mathrm{o}}+\frac{m g}{\mathrm{k}}
$$

By substituting Eq. (12) into Eq. (7) and Eq. (10), the capacitance for the first model:

$$
C=2 \pi \varepsilon_{\mathrm{o}} \frac{h-y_{\mathrm{o}}+\frac{m g}{\mathrm{k}}}{\ln \left(\frac{b}{a}\right)+\frac{1}{\kappa} \ln \left(\frac{c}{b}\right)}
$$

For the second model:

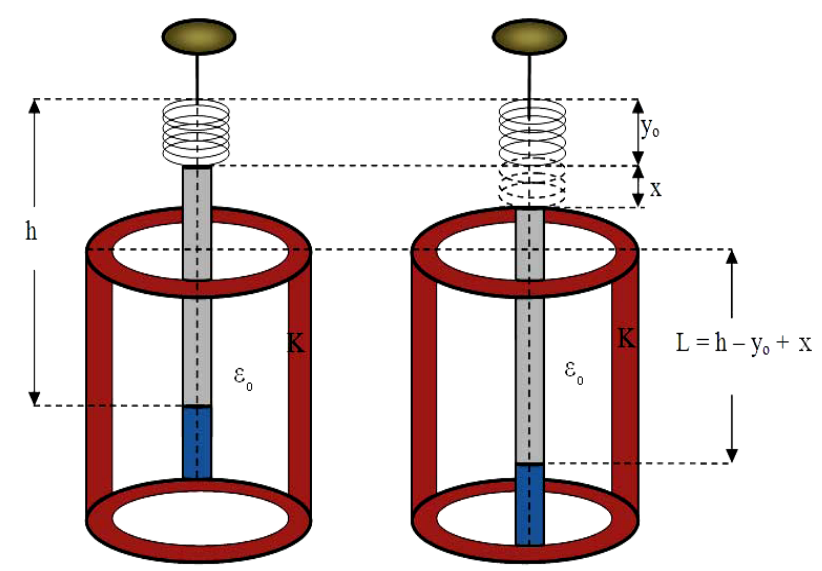

(a)

$$
C=2 \pi \varepsilon_{\mathrm{o}} \kappa_{\text {ravg }} \frac{h-y_{\mathrm{o}}+\frac{m g}{\mathrm{k}}}{\ln \left(\frac{b}{a}\right)}
$$

This gives us an expression for gravitational acceleration $(g)$ in terms of the capacitance values for the cylindrical capacitor. The first model, from Eq. (13):

$$
g=\frac{\mathrm{k}}{m}\left(\frac{C}{2 \pi \varepsilon_{\mathrm{o}}}\left(\ln \frac{b}{a}+\frac{1}{\kappa} \ln \frac{c}{b}\right)-h+y_{\mathrm{o}}\right)
$$

The second model; from Eq. (14):

$$
g=\frac{\mathrm{k}}{m}\left[\left(\frac{C}{2 \pi \varepsilon_{\mathrm{o}} \kappa_{\mathrm{ravg}}} \ln \frac{b}{a}\right)-h+y_{\mathrm{o}}\right]
$$

This gives us an expression for gravitational acceleration $(g)$ in terms of the capacitance $(C)$ between the cylindrical capacitor as well as the physical dimensions and properties of the system.

The structure of electrostatic cylindrical gravimeter is shown in Figs. 2a and 2b.

Comparisons between Fig. 2a and Fig. 2b are of both electrostatic gravimeter. The cylindrical, the spring of mass, and the hanging mass would all be constructed out of the same metallic material. The rest of the cylinder would be an insulating material. The capacitance meter would be attached to the top of the spring, and the bottom of the cylinder. A cylindrical geometry was chosen out of convenience.

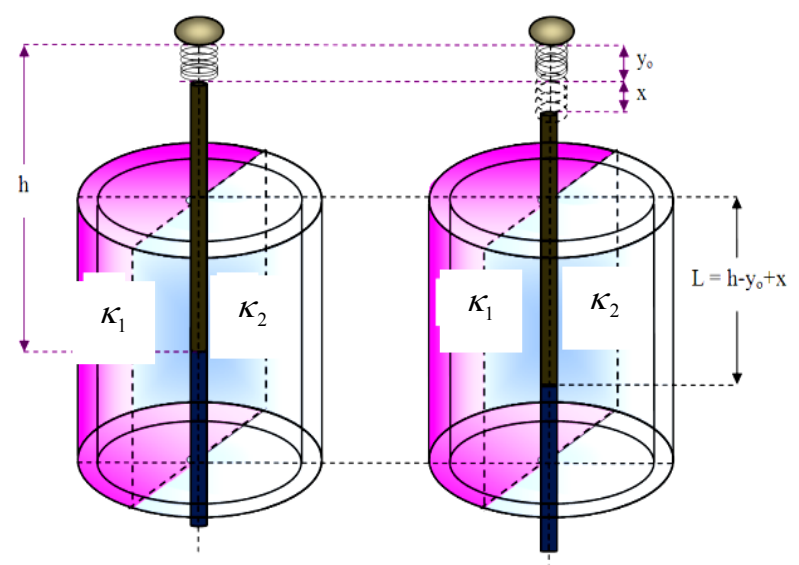

(b)

Fig. 1 Two models of electrostatic gravimeter, (a)1st and (b) 2nd model. 


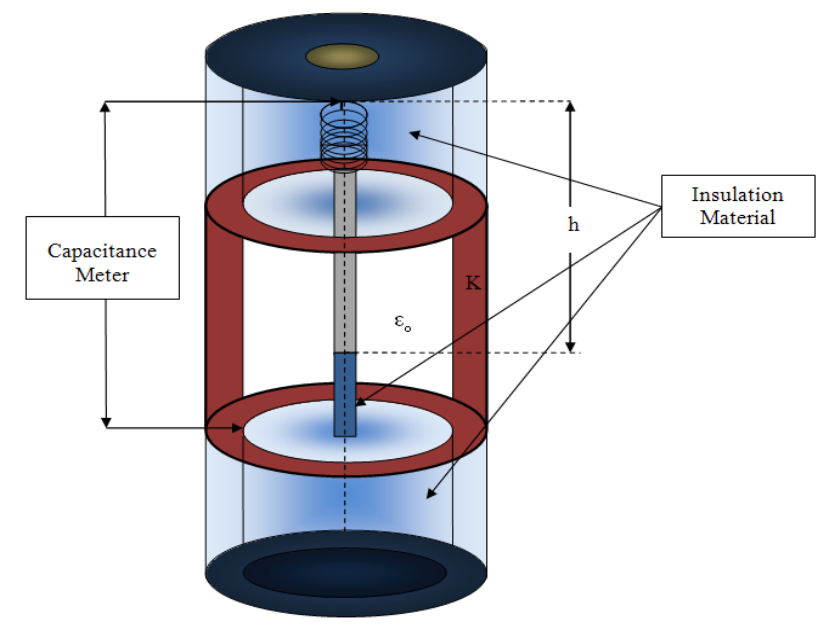

(a)

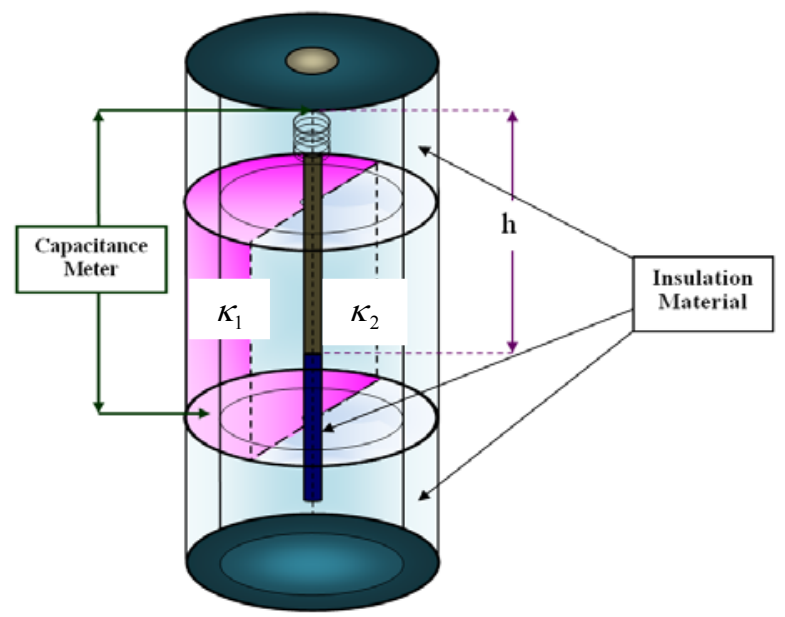

(b)

Fig. 2 The structure of our electrostatic cylindrical gravimeter, (a) 1st and (b) 2nd model.

\section{Results and Discussion}

In structure of electrostatics gravimeter, from study of the relationships between capacitance values for cylindrical capacitor and a system of sprinsg stretched from equilibrium position. It is called a new equipment of determining gravitational acceleration is "gravimeter capacitor". The process for obtaining capacitance for the cylindrical capacitor is simply a matter of reading a value given by a very precise meter, at various test position were carried out in the Ramkhamhaeng University. These data are presented in the Tables 1 and 2.

From the verifying of total changing of gravitational acceleration, we can then determine by plugging the appropriate values into Eq. (15) and Eq. (16). The material of your dielectric constant could be polyethylene, plexiglas and neoprene but for convenience, we chose to derive the neoprene using the dielectric constant. By looking at some realistic values, it can be shown that small changes in the gravitational acceleration produce changes in the capacitance that are easily measureable with a precise meter, as shown in Tables 3 and 4.

The experimental results from test by gravimeter capacitor are presented in Tables 3 and 4. The values may seem very exact, but an apparatus with these exact values can be easily manufactured by a skilled machinist [8]. Using these values, we find the range of
Table 1 Capacitance and gauging station value for the 1st model.

\begin{tabular}{lll}
\hline \multirow{2}{*}{$\begin{array}{l}\text { Height } \\
(\mathrm{m})\end{array}$} & \multicolumn{2}{c}{ Capacitance $(\mathrm{pF})$} \\
\cline { 2 - 3 } & Polyethylene & Neoprene \\
\hline 0.00 & $159.585119 \pm 0.001$ & $168.987967 \pm 0.001$ \\
2.98 & $159.584961 \pm 0.001$ & $168.987799 \pm 0.001$ \\
5.96 & $159.584801 \pm 0.001$ & $168.987630 \pm 0.001$ \\
8.94 & $159.584483 \pm 0.001$ & $168.987293 \pm 0.001$ \\
11.92 & $159.584324 \pm 0.001$ & $168.987124 \pm 0.001$ \\
14.89 & $159.584164 \pm 0.001$ & $168.986956 \pm 0.001$ \\
17.87 & $159.584005 \pm 0.001$ & $168.986787 \pm 0.001$ \\
20.85 & $159.583846 \pm 0.001$ & $168.986618 \pm 0.001$ \\
23.83 & $159.583686 \pm 0.001$ & $168.986450 \pm 0.001$ \\
26.81 & $159.583527 \pm 0.001$ & $168.986281 \pm 0.001$ \\
29.79 & $159.583209 \pm 0.001$ & $168.985944 \pm 0.001$ \\
\hline Range & $\Delta C_{1}=1.91 \times 10^{-3}$ & $\Delta C_{2}=2.023 \times 10^{-3}$ \\
\hline
\end{tabular}

Table 2 Capacitance and gauging station value for the 2st model.

\begin{tabular}{lll}
\hline \multirow{2}{*}{$\begin{array}{l}\text { Height } \\
(\mathrm{m})\end{array}$} & \multicolumn{2}{c}{ Capacitance $(\mathrm{pF})$} \\
\cline { 2 - 3 } & Polyethylene & Neoprene \\
\hline 0.00 & $301.913336 \pm 0.001$ & $556.156151 \pm 0.001$ \\
2.98 & $301.913035 \pm 0.001$ & $556.155596 \pm 0.001$ \\
5.96 & $301.912734 \pm 0.001$ & $556.155041 \pm 0.001$ \\
8.94 & $301.912131 \pm 0.001$ & $556.153931 \pm 0.001$ \\
11.92 & $301.911829 \pm 0.001$ & $556.153376 \pm 0.001$ \\
14.89 & $301.911529 \pm 0.001$ & $556.152821 \pm 0.001$ \\
17.87 & $301.911227 \pm 0.001$ & $556.152266 \pm 0.001$ \\
20.85 & $301.910926 \pm 0.001$ & $556.151711 \pm 0.001$ \\
23.83 & $301.910625 \pm 0.001$ & $556.151156 \pm 0.001$ \\
26.81 & $301.910323 \pm 0.001$ & $556.150601 \pm 0.001$ \\
29.79 & $301.909721 \pm 0.001$ & $556.149491 \pm 0.001$ \\
\hline Range & $\Delta C_{1}=3.615 \times 10^{-3}$ & $\Delta C_{2}=6.660 \times 10^{-3}$ \\
\hline
\end{tabular}




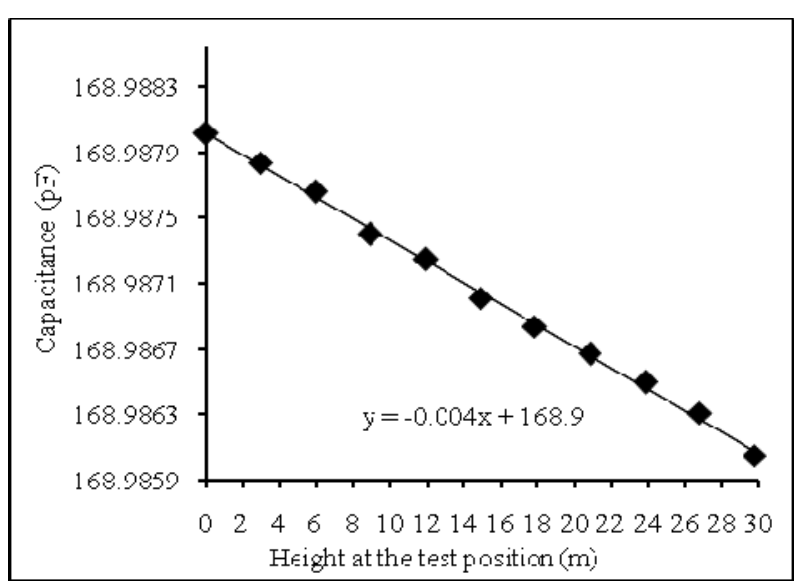

(a)

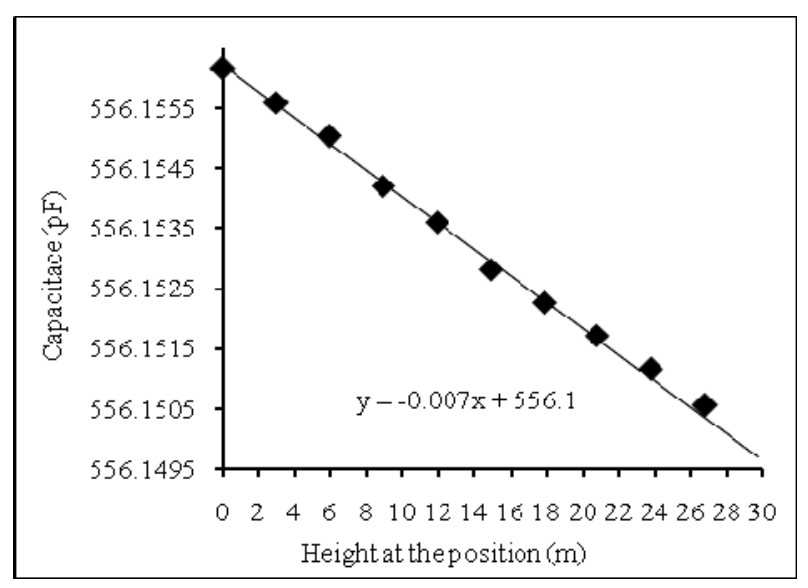

(b)

Fig. 3 The verifying change of capacitance at the test position, (a) the 1st model and (b) the 2st model.

Table 3 Capacitance and $g$ value for the 1st model.

\begin{tabular}{cll}
\hline $\begin{array}{l}\text { Height at the test } \\
\text { position }(\mathrm{m})\end{array}$ & $\begin{array}{l}\text { Capacitance } \\
(\mathrm{pF})\end{array}$ & $\begin{array}{l}\text { Gravitational } \\
\text { acceleration }\left(\mathrm{cm} / \mathrm{s}^{2}\right)\end{array}$ \\
\hline 0.00 & $168.987967 \pm 0.001$ & $978.312 \pm 0.001$ \\
2.98 & $168.987799 \pm 0.001$ & $978.311 \pm 0.001$ \\
5.96 & $168.987630 \pm 0.001$ & $978.310 \pm 0.001$ \\
8.94 & $168.987293 \pm 0.001$ & $978.308 \pm 0.001$ \\
11.92 & $168.987124 \pm 0.001$ & $978.307 \pm 0.001$ \\
14.89 & $168.986956 \pm 0.001$ & $978.306 \pm 0.001$ \\
17.87 & $168.986787 \pm 0.001$ & $978.305 \pm 0.001$ \\
20.85 & $168.986618 \pm 0.001$ & $978.304 \pm 0.001$ \\
23.83 & $168.986450 \pm 0.001$ & $978.303 \pm 0.001$ \\
26.81 & $168.986281 \pm 0.001$ & $978.302 \pm 0.001$ \\
29.79 & $168.985944 \pm 0.001$ & $978.300 \pm 0.001$ \\
\hline Range & $\Delta C=2.023 \times 10^{-3}$ & $\Delta g=0.012$ \\
\hline
\end{tabular}

Table 4 Capacitance and $g$ value for the 2 st model.

\begin{tabular}{cll}
\hline $\begin{array}{l}\text { Height at the test } \\
\text { position }(\mathrm{m})\end{array}$ & $\begin{array}{l}\text { Capacitance } \\
(\mathrm{pF})\end{array}$ & $\begin{array}{l}\text { Gravitational } \\
\text { acceleration }\left(\mathrm{cm} / \mathrm{s}^{2}\right)\end{array}$ \\
\hline 0.00 & $556.156151 \pm 0.001$ & $978.312 \pm 0.001$ \\
2.98 & $556.155596 \pm 0.001$ & $978.311 \pm 0.001$ \\
5.96 & $556.155041 \pm 0.001$ & $978.310 \pm 0.001$ \\
8.94 & $556.153931 \pm 0.001$ & $978.308 \pm 0.001$ \\
11.92 & $556.153376 \pm 0.001$ & $978.307 \pm 0.001$ \\
14.89 & $556.152821 \pm 0.001$ & $978.306 \pm 0.001$ \\
17.87 & $556.152266 \pm 0.001$ & $978.305 \pm 0.001$ \\
20.85 & $556.151711 \pm 0.001$ & $978.304 \pm 0.001$ \\
23.83 & $556.151156 \pm 0.001$ & $978.303 \pm 0.001$ \\
26.81 & $556.150601 \pm 0.001$ & $978.302 \pm 0.001$ \\
29.79 & $556.149491 \pm 0.001$ & $978.300 \pm 0.001$ \\
\hline Range & $\Delta C=6.660 \times 10^{-3}$ & $\Delta g=0.012$ \\
\hline
\end{tabular}

$\mathrm{C}$ to be approximately $168.985944 \mathrm{pF}-168.987967 \mathrm{pF}$ for the 1 st model and $556.149491 \mathrm{pF}-556.156151 \mathrm{pF}$ for the 2 nd model. To measure changes in gravitational acceleration on reference point, we need to detect changes in capacitance of $\Delta C_{1}=2.023 \times 10^{-3}$ and $\Delta C_{2}=$ $6.660 \times 10^{-3}$. In the field of electronics, there are meters that can easily detect changes in capacitance on the order of $1.00 \times 10^{-14}$, as shown in Figs. 3a-3b.

The values of gravitational acceleration calculated from capacitance as a function of height at various positions are shown in Fig. 4.

From the best fit shown in Fig. 4, it can easily be shown that a change in height of approximately $1 \mathrm{~m}$ results in a change in gravitational acceleration of $\Delta g=$ $-0.0031 \mathrm{~cm} / \mathrm{s}^{2}[9,10]$. From this result, we can infer that one should easily be able to detect the change in gravitational acceleration that occurs when moving between two floors of the test position.

\section{Conclusions}

Design for gravimeter by incorporating a mass spring system with a capacitor, but alters the method of determining the change in position of the mass attached to the spring caused by gravitational acceleration. We relate the change in position of mass to the capacitance for the cylindrical capacitor, and obtain the relation between gravitational and the change in position; $g=$ $-0.003 x+978.310 \mathrm{~cm} / \mathrm{s}^{2}$, whereas the reference point of Ramkhamhaeng University $g=978.310 \mathrm{~cm} / \mathrm{s}^{2}$.

Absolute gravity measurements are most sensitive to 


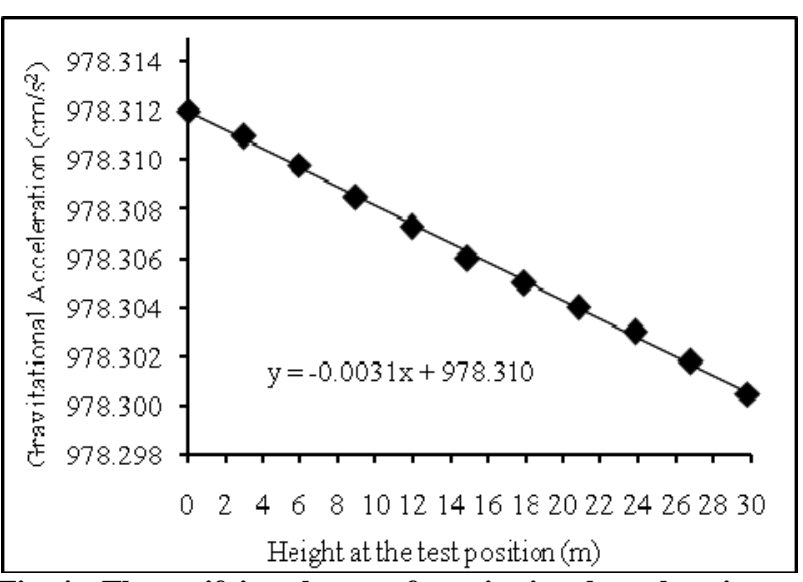

Fig. 4 The verifying change of gravitational acceleration at the test position.

height changes and provide an obvious way to define and control the vertical height datum. No additional reference points at the Earth's surface and no observations of celestial bodies or satellites are needed. Shortcomings of relative gravimeter, like calibration problems and deficiencies in the datum level definition, can be overcome. The accuracy of an absolute gravity net is independent of geographical extension which allows applications on local, regional and global scales with consistent measurement quality. A combination of gravimetric and geometric measurements may enable discrimination among subsurface mass movements associated with or without a surface deformation.

\section{References}

[1] R.A. Serway, J.W. Jewett, Physics for Scientists and Engineers, 6th ed., Thomson Learning Inc. California State Polytechnic University, Pomona, 2004, pp. 389-441.

[2] J.P. Davidson, W.E. Reed, P.M. Davis, Exploring Earth, 2nd ed., Prentice Hall, New Jersey, 2002, pp. 135-142.

[3] R.J. Lillie, Whole Earth Geophysics, Prentice Hall, New Jersey, 1999, pp. 223-275.

[4] G.R. Fowles, G.L. Cassiday, Analytical Mechanics 6th ed., Thomson Learning Inc., 1999, pp. 79-80.

[5] W.F. Hoffmann, A pendulum gravimeter for measurement of periodic annual variations in the gravitational constant, Ph.D., Thesis, Princeton University, 1962.

[6] A.E. Mussett, M.A. Khan, Looking into the Earth: An Introduction to Geological Geophysics. Cambridge (Cambridge University Press), 2000, pp. 107-123.

[7] D.J. Griffiths, Introduction to Electrodynamics, 3rd Ed., Prentice Hall, New Jersey, 1999, pp. 103-106, 179-196.

[8] D. Halliday, R. Resnick, J. Walker, Fundamentals of Physics, 7th ed., John Wiley \& Sons, Inc., New London, Connecticut, 2005, pp. 24-27.

[9] D.O. Kashinski, P.V. Quinn, The electrostatic gravimeter: An alternate way of measuring gravitational acceleration. Master, Thesis, University of Kutztown, Pennsylvania, 2006.

[10] P. Mahapattanathai, W. Ngansom, Gravimeter: The cylindrical capacitor two dielectrics, in: International Conference 37th Congress on Science and Technology of Thailand, 2011, pp. 64. 